\title{
Effects of Electric Field and Poling on the Mode I Energy Release Rate for Two Symmetric Edge Cracks in Rectangular Piezoelectric Ceramic Strips*
}

\author{
Yasuhide SHINDO**, Fumio NARITA** and Takuya MATSUDA** \\ ** Department of Materials Processing, Graduate School of Engineering, Tohoku University, \\ Aoba-yama 6-6-02, Sendai 980-8579, Japan \\ E-mail: shindo@material.tohoku.ac.jp
}

\begin{abstract}
This article discusses the results of the mode I energy release rate of rectangular piezoelectric ceramic strips with two symmetric edge cracks under electromechanical loading. Cracks were created normal or parallel to the poling direction, and applied electric fields were parallel/antiparallel to the poling. A nonlinear plane strain finite element analysis was performed, and the effect of localized polarization switching on the energy release rate was discussed for the permeable, impermeable, open and discharging cracks under a high negative electric field. In the case under a high positive electric field, we examined the effect of dielectric breakdown on the energy release rate.
\end{abstract}

Key words : Crack Mechanics; Finite Element Method; Piezoelectric Ceramics; Energy Release Rate; Smart Materials and Structures

\section{Introduction}

The study of mechanical behavior in piezoelectric lead zirconate titanate (PZT) ceramics offer important considerations in the design of smart materials and structures. In the theoretical studies of the piezoelectric crack problems, the electrical boundary condition across the crack face remains a debating issue. There are three commonly used electrical boundary conditions, i.e. the permeable $\operatorname{crack}^{(1)}$, impermeable $\operatorname{crack}^{(2),(3)}$ and open piezoelectric $\mathrm{crack}^{(4)}$ models. Although the impermeable and open crack models may provide the mathematical solutions of the piezoelectric cracks, it is still questionable to use these models. Recently, Landis ${ }^{(5)}$ proposed a non-linear electrically discharging crack model and discussed the effect of electric breakdown field within the crack gap on the energy release rate of the piezoelectric ceramics. Mode I stress and electric displacement were applied, and various levels of discharge field within the crack gap were used. However, doubt remained as to whether these conditions would be suitable, that is, the electric displacement can usually not be applied to the piezoelectric ceramics and most of the discharge levels are very high. In order to resolve the effect of the crack face electrical boundary condition on the piezoelectric fracture mechanics parameters, Shindo et al. ${ }^{(6)}$ discussed the mode I energy release rate of the piezoelectric ceramics under electromechanical loading. They showed that, in modeling cracks in piezoelectric ceramics, the impermeable and open crack face assumptions can lead to unphysical negative energy release rate under positive and negative electric fields and produce significant errors. They also found that using the standard air breakdown field for a critical discharge level within the crack gap, the energy release rate for the discharging crack model becomes the energy release rate for the permeable crack model.

Some works ${ }^{(7),(8)}$ showed that under a high negative electric field, the piezoelectric crack behavior is influenced by the polarization switching. It is also expected that the dielectric breakdown under a high positive electric field affect the fracture behavior of piezoelectric

*Received 21 Feb., 2011 (No. 11-0126) [DOI: 10.1299/jmmp.5.335]

Copyright (c) 2011 by JSME 


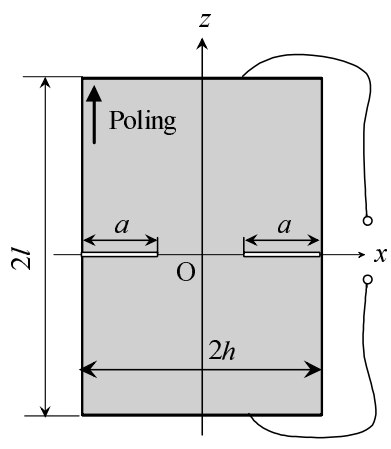

(a)

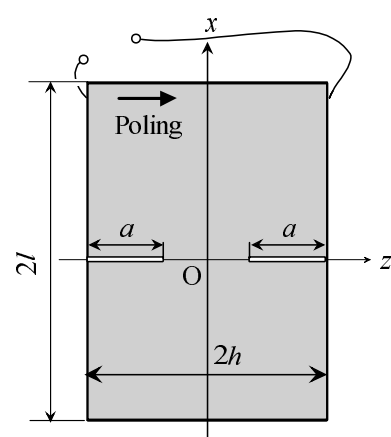

(b)

Fig. 1 Rectangular piezoelectric ceramic strips with two symmetric edge cracks: (a) Case 1; (b) Case 2

ceramics ${ }^{(9)}$. Although a numerical method for the polarization saturation ${ }^{(10)} /$ strip dielectric breakdown ${ }^{(11)}$ model was proposed for cracked piezoelectric ceramics, the impermeable and open crack face assumptions and unrealistic loading conditions (very high applied stress and electric field/electric displacement) were used to calculate the energy release rate ${ }^{(12)}$, and meaningless results were given.

The purpose of this work is to investigate the effect of electromechanical loading on the mode I energy release rate of rectangular piezoelectric ceramic strips containing two symmetric edge cracks normal to the edges of the strip. Cracks were created normal or parallel to the poling direction, and electric fields were applied parallel/antiparallel to the poling. The mode I energy release rate was calculated by the nonlinear finite element method for the plane strain permeable, impermeable, open and discharging crack models. Electric field dependence of the energy release rate was then discussed under various crack face electrical boundary conditions. The effects of localized polarization switching and dielectric breakdown on the energy release rate were also examined.

\section{Finite element solution procedure}

\subsection{Problem statement and basic equations}

Consider rectangular piezoelectric ceramic strips of length $2 l$ and width $2 h$ which contain two symmetric edge cracks of length $a$. The crack is assumed with faces normal (Case 1) or parallel (Case 2) to the polarization axis, as shown in Fig.1. Let $x, y$ and $z$ denote the Cartesian coordinates. We assume that the piezoelectric ceramic strips poled in the $z$-direction are in plane strain state in the $y$-direction, and are loaded by mechanical tension and electric field. Usually the piezoelectric materials are driven by poling direction electric field and so we consider here the piezoelectric material subjected to electric field parallel/untiparallel to the poling direction ( $z$-axis). Because of symmetry, only the first quadrant with appropriate boundary conditions needs to be analyzed.

The governing equations are obtained as

$$
\begin{aligned}
& \left.\begin{array}{l}
\sigma_{x x, x}+\sigma_{z x, z}=0 \\
\sigma_{x z, x}+\sigma_{z z, z}=0
\end{array}\right\} \\
& D_{x, x}+D_{z, z}=0
\end{aligned}
$$

where $\sigma_{x x}, \sigma_{z z}, \sigma_{x z}=\sigma_{z x}$ are the components of stress tensor, $D_{x}$ and $D_{z}$ are the components of electric displacement vector, and a comma implies partial differentiation with respect to the coordinates. The constitutive equations are given by

$$
\left\{\begin{array}{c}
\sigma_{x x} \\
\sigma_{z z} \\
\sigma_{z x}
\end{array}\right\}=\left[\begin{array}{ccc}
c_{11} & c_{13} & 0 \\
c_{13} & c_{33} & 0 \\
0 & 0 & c_{44}
\end{array}\right]\left\{\begin{array}{c}
\varepsilon_{x x}-\varepsilon_{x x}^{\mathrm{r}} \\
\varepsilon_{z z}-\varepsilon_{z z}^{\mathrm{r}} \\
2\left(\varepsilon_{z x}-\varepsilon_{z x}^{\mathrm{r}}\right)
\end{array}\right\}-\left[\begin{array}{cc}
0 & e_{31} \\
0 & e_{33} \\
e_{15} & 0
\end{array}\right]\left\{\begin{array}{c}
E_{x} \\
E_{z}
\end{array}\right\}
$$




$$
\begin{aligned}
\left\{\begin{array}{c}
D_{x} \\
D_{z}
\end{array}\right\}=\left[\begin{array}{ccc}
0 & 0 & e_{15} \\
e_{31} & e_{33} & 0
\end{array}\right]\left\{\begin{array}{c}
\varepsilon_{x x}-\varepsilon_{x x}^{\mathrm{r}} \\
\varepsilon_{z z}-\varepsilon_{z z}^{\mathrm{r}} \\
2\left(\varepsilon_{z x}-\varepsilon_{z x}^{\mathrm{r}}\right)
\end{array}\right\} \\
+\left[\begin{array}{cc}
\epsilon_{11} & 0 \\
0 & \epsilon_{33}
\end{array}\right]\left\{\begin{array}{c}
E_{x} \\
E_{z}
\end{array}\right\}+\left\{\begin{array}{c}
P_{x}^{\mathrm{r}} \\
P_{z}^{\mathrm{r}}
\end{array}\right\}
\end{aligned}
$$

where $\varepsilon_{x x}, \varepsilon_{z z}, \varepsilon_{z x}$ are the components of strain tensor, $E_{x}$ and $E_{z}$ are the components of electric field intensity vector, $c_{11}, c_{13}, c_{33}, c_{44}$ are the elastic stiffness constants, $\epsilon_{11}, \epsilon_{33}$ are the dielectric permittivities, and $e_{15}, e_{31}, e_{33}$ are the piezoelectric constants. In Eqs.(3), (4), $\varepsilon_{x x}^{\mathrm{r}}, \varepsilon_{z z}^{\mathrm{r}}, \varepsilon_{z x}^{\mathrm{r}}$ and $P_{x}^{\mathrm{r}}, P_{z}^{\mathrm{r}}$ are the components of remanent strain tensor and remanent polarization vector, respectively. The relation between the components of strain tensor and the components of displacement vector, $u_{x}$ and $u_{z}$, are given by

$$
\varepsilon_{x x}=u_{x, x}, \quad \varepsilon_{z z}=u_{z, z}, \quad \varepsilon_{x z}=\varepsilon_{z x}=\left(u_{z, x}+u_{x, z}\right) / 2
$$

The components of electric field vector are related to the gradient of the electric potential $\phi(x, z)$ by

$$
E_{x}=-\phi_{, x}, \quad E_{z}=-\phi_{, z}
$$

With Eqs. (3)-(6), the governing equations can be written as

$$
\left.\begin{array}{l}
c_{11} u_{x, x x}+c_{44} u_{x, z z}+\left(c_{13}+c_{44}\right) u_{z, x z}+\left(e_{31}+e_{15}\right) \phi_{, x z}=0 \\
c_{44} u_{z, x x}+c_{33} u_{z, z z}+\left(c_{13}+c_{44}\right) u_{x, x z}+e_{15} \phi_{, x x}+e_{33} \phi_{, z z}=0
\end{array}\right\}
$$

In a vacuum, the constitutive equations (4) and the governing equation (8) become

$$
\begin{aligned}
& D_{x}=\epsilon_{0} E_{x}, \quad D_{z}=\epsilon_{0} E_{z} \\
& \phi_{, x x}+\phi_{, z z}=0
\end{aligned}
$$

where $\epsilon_{0}=8.85 \times 10^{-12} \mathrm{C} / \mathrm{Vm}$ is the dielectric permittivity of the vacuum.

We first consider the case 1 (see Fig. 1(a)). The boundary conditions at $z=0$ can be written as

$$
\left.\begin{array}{l}
\sigma_{z x}(x, 0)=0 \quad(0 \leq x \leq h) \\
\left.\begin{array}{l}
u_{z}(x, 0)=0 \quad(0 \leq x \leq h-a) \\
\sigma_{z z}(x, 0)=0 \quad(h-a<x \leq h)
\end{array}\right\} \\
\phi(x, 0)=0 \quad(0 \leq x \leq h-a) \\
E_{x}(x, 0)=E_{x}^{c}(x, 0) \quad(h-a<x \leq h)
\end{array}\right\}
$$

where the superscript $c$ stands for the electric field quantity in the void inside the crack. The electric potentials $\phi(x, z), \phi^{c}(x, z)$ and electric field intensities $E_{x}(x, z), E_{x}^{c}(x, z)$ are odd functions of $z$. The electric field intensity $E_{x}^{c}(x, 0)$ is zero on the symmetry plane inside the crack and the $E_{x}(x, 0)$ is equal to zero ahead of the crack, so the boundary conditions of Eqs. (13) reduce to $E_{x}(x, 0)=0(0 \leq x \leq h)$. The condition $E_{x}(x, 0)=0(0 \leq x \leq h)$ is equivalent to the condition $\phi(x, 0)=0(0 \leq x \leq h)$. Eqs. (13) and (14) are the permeable boundary conditions. The loading conditions are

$$
u_{z}(x, l)=u_{0}, \phi(x, l)=\phi_{0} \quad(0 \leq x \leq h)
$$




$$
\sigma_{x x}(h, z)=0, \sigma_{x z}(h, z)=0, D_{x}(h, z)=0(0 \leq z \leq l)
$$

where $u_{0}$ is the applied displacement and $\phi_{0}$ is the applied electric potential. In electrostatics, at a surface separating two dielectric mediums, the normal component of the electric displacement and the tangential component of the electric field are continuous. However, when one of the mediums is air, these two conditions can be approximated simply by one, namely that the normal component of the electric displacement vanishes at the surface. So the third of Eq. (16) is adopted here. This assumption is based on the fact that there is a very large difference between the dielectric constants of the piezoelectric material and the air ${ }^{(13)}$. The electric displacement $D_{z}(x, 0)$ is solved under the crack face boundary (Eqs. (11)-(13)) and loading (Eqs. (15), (16)) conditions by the finite element analysis, and the $D_{z}^{c}(x, 0)$ is determined by Eq. (14) precisely.

On the other hand, the impermeable boundary condition is

$$
\left.\begin{array}{l}
\phi(x, 0)=0 \quad(0 \leq x \leq h-a) \\
D_{z}(x, 0)=0 \quad(h-a<x \leq h)
\end{array}\right\}
$$

The condition $D_{z}(x, 0)=0(h-a<x \leq h)$ is based on the fact that there is a very large difference between the dielectric constants of the piezoelectric material and the void inside the crack. However, it will be shown that this condition is not appropriate for a slit crack in the piezoelectric materials. Also, the electrical boundary condition for the open crack model is given by

$$
\left.\begin{array}{l}
\phi(x, 0)=0 \quad(0 \leq x \leq h-a) \\
D_{z}^{+}=D_{z}^{-} \quad(h-a<x \leq h) \\
D_{z}^{+}\left(u_{z}^{+}-u_{z}^{-}\right)=\epsilon_{0}\left(\phi^{-}-\phi^{+}\right) \quad(h-a<x \leq h)
\end{array}\right\}
$$

where the superscripts + and - denote the upper and lower crack faces, respectively.

By applying the loading conditions (15), the stress $\sigma_{z z}$ for the uncracked piezoelectric material is obtained as

$$
\sigma_{z z}(x, z)=\sigma_{0}-e_{1} E_{0}
$$

where

$$
\begin{aligned}
& \sigma_{0}=\left(c_{33}-\frac{c_{13}^{2}}{c_{11}}\right) \varepsilon_{0} \\
& \varepsilon_{0}=\frac{u_{0}}{l}, \quad E_{0}=-\frac{\phi_{0}}{l}
\end{aligned}
$$

and

$$
e_{1}=e_{33}-\left(\frac{c_{13}}{c_{11}}\right) e_{31}
$$

The stress at $z=l$ for the uncracked piezoelectric material is denoted by $\sigma_{l}=\sigma_{0}-e_{1} E_{0}$. Note that $\sigma_{0}$ is the stress for a closed-circuit condition with the potential forced to remain zero (grounded).

Next, we consider the case 2 (see Fig. 1(b)). The boundary conditions at $x=0$ are given by

$$
\left.\begin{array}{l}
\sigma_{x z}(0, z)=0 \quad(0 \leq z \leq h) \\
\left.\begin{array}{l}
u_{x}(0, z)=0 \quad(0 \leq z \leq h-a) \\
\sigma_{x x}(0, z)=0 \quad(h-a<z \leq h)
\end{array}\right\} \\
\phi_{, x}(0, z)=0 \quad(0 \leq z \leq h-a) \\
E_{z}(0, z)=E_{z}^{c}(0, z) \quad(h-a<z \leq h)
\end{array}\right\}
$$




$$
D_{x}(0, z)=D_{x}^{c}(0, z) \quad(h-a<z \leq h)
$$

The loading conditions are

$$
\begin{aligned}
& u_{x}(l, z)=u_{0}, \quad D_{x}(l, z)=0 \quad(0 \leq z \leq h) \\
& \sigma_{z z}(x, h)=0, \quad \sigma_{z x}(x, h)=0, \quad \phi(x, h)=\phi_{0} \quad(0 \leq x \leq l)
\end{aligned}
$$

The electric potential is zero on the $z=0$ plane (symmetry plane). Equations (25) and (26) are the permeable boundary conditions. We also consider the electrical boundary conditions for the impermeable and open crack models.

By applying the loading conditions, the stress $\sigma_{x x}$ for the uncracked piezoelectric material is obtained as

$$
\sigma_{x x}(x, z)=c_{11} \varepsilon_{0}
$$

The stress at $x=l$ for the uncracked piezoelectric material is given by $\sigma_{l}=c_{11} \varepsilon_{0}$.

\subsection{Polarization switching}

A non-linear finite element model incorporating the polarization switching mechanisms is presented. We assumed that for $90^{\circ}$ switching there are two allowable directions of the poling in the coordinate system: in the positive and negative $x$-direction. Two criteria are considered. The first criterion (work done switching criterion) requires that a polarization switches when the combined electrical and mechanical work exceeds a critical value ${ }^{(14)}$, i.e.

$$
\sigma_{x x} \Delta \varepsilon_{x x}+\sigma_{z z} \Delta \varepsilon_{z z}+2 \sigma_{z x} \Delta \varepsilon_{z x}+E_{x} \Delta P_{x}+E_{z} \Delta P_{z} \geq 2 P^{\mathrm{s}} E_{\mathrm{c}}
$$

where $\Delta \varepsilon_{x x}, \Delta \varepsilon_{z z}, \Delta \varepsilon_{z x}$ are the changes in the spontaneous strain $\gamma^{\mathrm{s}}, \Delta P_{x}, \Delta P_{z}$ are the changes in the spontaneous polarization $P^{\mathrm{s}}$ (see Appendix A), and $E_{\mathrm{c}}$ is a coercive electric field. It is assumed that elastic and dielectric constants of the piezoelectric materials remain unchanged after $180^{\circ}$ or $90^{\circ}$ polarization switching occurs and only piezoelectric constants vary with switching. The second criterion (internal energy density criterion) is defined as ${ }^{(15)}$

$$
U \geq U_{\mathrm{c}}
$$

where $U$ is the internal energy density and $U_{c}$ is a critical value of internal energy density corresponding to the switching mode. The internal energy density associated with $180^{\circ}$ switching can be expressed as

$$
U=\frac{1}{2} D_{z} E_{z}
$$

In the case of $90^{\circ}$ switching, the internal energy density is

$$
U=\frac{1}{2}\left(\sigma_{x x} \varepsilon_{x x}+\sigma_{z z} \varepsilon_{z z}+2 \sigma_{z x} \varepsilon_{z x}+D_{x} E_{x}\right)
$$

The critical value of internal energy density is assumed in the following form:

$$
U_{\mathrm{c}}=\frac{1}{2} \epsilon_{33}^{\mathrm{T}}\left(E_{\mathrm{c}}\right)^{2}
$$

where $\epsilon_{33}^{\mathrm{T}}$ is the dielectric permittivity at constant stress. Plane strain condition is held after $180^{\circ}$ and $90^{\circ}$ switching, and the constitutive equations (3) and (4) after switching are given in Appendix B.

\subsection{Dielectric breakdown}

For the piezoelectric ceramics under high positive electric fields, the material will break down electrically. A very simple model is used here for such electrical discharges. It is assumed that the material cannot support electric fields larger than the breakdown strength $E_{d}$. That is, the electric field in the dielectric breakdown region is equal to $E_{d}$. 


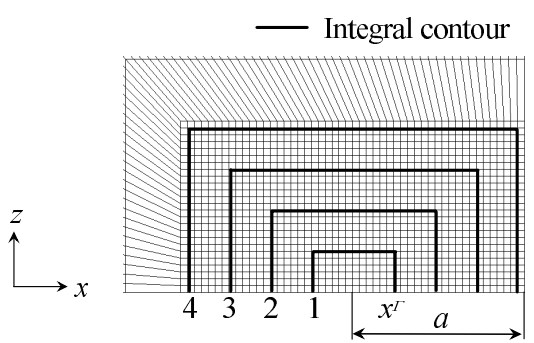

Fig. 2 Finite element mesh and boundaries of integration domains

\subsection{Energy release rate}

The energy release rate $G$ for the piezoelectric materials can be obtained by ${ }^{(8)}$ :

$$
\begin{aligned}
G= & \left(\int_{\Gamma_{0}}-\int_{\Gamma_{\mathrm{p}}}\right)\left\{H n_{x}-\left(\sigma_{x x} u_{x, x}+\sigma_{z x} u_{z, x}\right) n_{x}-\left(\sigma_{z x} u_{x, x}+\sigma_{z z} u_{z, x}\right) n_{z}\right. \\
& \left.+D_{x} E_{x} n_{x}+D_{z} E_{x} n_{z}\right\} d \Gamma
\end{aligned}
$$

where $H$ is an electrical enthalpy density, $\Gamma_{0}$ is a small contour closing a crack tip, $\Gamma_{\mathrm{p}}$ is a path embracing that part of phase boundary which is enclosed by $\Gamma_{0}$, and $n_{x}, n_{z}$ are the components of the outer unit normal vector.

The $G$ for the permeable and impermeable crack models are path-independent. If the crack gap can store energy, nonzero contributions to $G$ for the open crack model arise from the contour segments along the crack face ${ }^{(16)}$. The values of $G$ for the open crack model can be obtained by computing contour integrations and then subtracting the electrical enthalpy density of the crack gap $H^{c}$ times the crack opening displacement evaluated at the intersection $x^{\Gamma}$ of the contour with the crack faces ${ }^{(5)}$. So Eq. (35) becomes

$$
\begin{aligned}
G= & \left(\int_{\Gamma_{0}}-\int_{\Gamma_{\mathrm{p}}}\right)\left\{H n_{x}-\left(\sigma_{x x} u_{x, x}+\sigma_{z x} u_{z, x}\right) n_{x}-\left(\sigma_{z x} u_{x, x}+\sigma_{z z} u_{z, x}\right) n_{z}\right. \\
& \left.+D_{x} E_{x} n_{x}+D_{z} E_{x} n_{z}\right\} d \Gamma-2 H^{c}\left(x^{\Gamma}\right) u_{z}^{+}\left(x^{\Gamma}\right)
\end{aligned}
$$

The values of $G$ for the non-linear electrically discharging crack model can be obtained by using Eq. (36) and assuming that the crack gap behaves in a linear dielectric manner when electric field within crack gap is below a critical discharge level $E_{d}^{c(5)}$.

Plane strain finite element analysis was carried out to evaluate the energy release rates for the permeable, impermeable, open and discharging crack models using the domain integral $\operatorname{method}^{(17)}$, and for the calculation of $G$, four domains were defined in the finite element mesh. Fig. 2 gives the detail of crack tip mesh for Case 1. The $G$ calculations for the open and discharging crack models are complicated than for the permeable and impermeable crack models ${ }^{(18)}$. The Newton-Raphson method was used to find the solutions for the open and discharging crack models.

The polarization switching is defined for each element. The displacement $u_{0}$ and electric potential $\phi_{0}$ are applied, and the electromechanical fields of each element are computed from the finite element analysis. The switching criterion of Eq. (30) or (31) is checked for every element to see if switching will occur. After all possible polarization switches have occurred, the piezoelectric tensor of each element is rotated to the new polarization direction, and the electromechanical fields are recalculated. Such a procedure is repeated until the evolution of the objective solutions shows no improvements. The possible occurrence of dielectric breakdown is also assessed for every element.

The spontaneous polarization $P^{\mathrm{s}}$ and strain $\gamma^{\mathrm{s}}$ are assigned representative values of 0.3 $\mathrm{C} / \mathrm{m}^{2}$ and 0.004 , respectively. Our previous experiments ${ }^{(19),(20)}$ verified the accuracy of the above scheme, and showed that the obtained results are of general applicability. 
Table 1 Material properties of PZTs

\begin{tabular}{lccccccccc}
\hline & \multicolumn{3}{c}{$\begin{array}{c}\text { Elastic stiffnesses } \\
\left(\times 10^{10} \mathrm{~N} / \mathrm{m}^{2}\right)\end{array}$} & \multicolumn{3}{c}{$\begin{array}{c}\text { Piezoelectric coefficients } \\
\left(\mathrm{C} / \mathrm{m}^{2}\right)\end{array}$} & \multicolumn{2}{c}{$\begin{array}{c}\text { Dielectric constants } \\
\left(\times 10^{-10} \mathrm{C} / \mathrm{Vm}\right)\end{array}$} \\
\cline { 2 - 10 } & $c_{11}$ & $c_{33}$ & $c_{44}$ & $c_{13}$ & $e_{31}$ & $e_{33}$ & $e_{15}$ & $\epsilon_{11}$ & $\epsilon_{33}$ \\
\hline PCM-80 & 17.0 & 16.5 & 3.05 & 11.5 & -5.99 & 15.6 & 13.7 & 95.2 & 68.4 \\
C-91 & 12.0 & 11.4 & 2.4 & 7.7 & -17.3 & 21.2 & 20.2 & 226 & 235 \\
\hline
\end{tabular}

Table 2 Energy release rate for rectangular piezoelectric material PCM-80 under $u_{0}=1 \mu \mathrm{m}$ and $E_{0}=0.1$ $\mathrm{MV} / \mathrm{m}$ (Case 1)

\begin{tabular}{|c|c|c|c|c|}
\hline \multirow{2}{*}{$\begin{array}{r}\text { PCM-80 } \\
\text { Case } 1\end{array}$} & \multicolumn{4}{|c|}{$G\left(\mathrm{~N} / \mathrm{m}^{2}\right)$} \\
\hline & Permeable & Impermeable & Open & Discharging \\
\hline \multicolumn{5}{|l|}{ Domain } \\
\hline 1 & 4.835 & 4.323 & $4.816\left(-1.1 \times 10^{-2}\right)$ & $4.835\left(-1.3 \times 10^{-5}\right)$ \\
\hline 2 & 4.827 & 4.315 & $4.799\left(-1.4 \times 10^{-2}\right)$ & $4.827\left(-1.6 \times 10^{-5}\right)$ \\
\hline 3 & 4.820 & 4.309 & $4.785\left(-1.6 \times 10^{-2}\right)$ & $4.820\left(-1.8 \times 10^{-5}\right)$ \\
\hline 4 & 4.815 & 4.304 & $4.773\left(-1.7 \times 10^{-2}\right)$ & $4.814\left(-2.0 \times 10^{-5}\right)$ \\
\hline Avg. & 4.824 & 4.313 & $4.793\left(-1.5 \times 10^{-2}\right)$ & $4.824\left(-1.7 \times 10^{-5}\right)$ \\
\hline \multicolumn{5}{|l|}{ Line } \\
\hline 1 & 4.835 & 4.323 & $4.816\left(-1.1 \times 10^{-2}\right)$ & $4.835\left(-1.3 \times 10^{-5}\right)$ \\
\hline 2 & 4.818 & 4.306 & $4.782\left(-1.6 \times 10^{-2}\right)$ & $4.826\left(-1.9 \times 10^{-5}\right)$ \\
\hline 3 & 4.807 & 4.297 & $4.758\left(-2.0 \times 10^{-2}\right)$ & $4.820\left(-2.3 \times 10^{-5}\right)$ \\
\hline 4 & 4.798 & 4.292 & $4.739\left(-2.3 \times 10^{-2}\right)$ & $4.814\left(-2.6 \times 10^{-5}\right)$ \\
\hline Avg. & 4.814 & 4.304 & $4.774\left(-1.7 \times 10^{-2}\right)$ & $4.814\left(-2.0 \times 10^{-5}\right)$ \\
\hline
\end{tabular}

Values in parentheses are the results of $2 H^{c}\left(x^{\Gamma}\right) u_{z}^{+}\left(x^{\Gamma}\right)$ in Eq. (36)

\section{Numerical results and discussion}

Numerical calculations have been carried out for commercially available piezoelectric ceramics, hard PZT PCM-80 (Panasonic Electric Devices Co., Ltd., Japan) and soft PZT C-91 (Fuji Ceramics Co., Ltd., Japan). The material properties are listed in Table 1, and the coercive electric fields of PCM-80 and C-91 are approximately $E_{c}=2$ and $0.35 \mathrm{MV} / \mathrm{m}$, respectively. The crack length is $a=5 \mathrm{~mm}$, and the ceramic width and length are $2 h=20 \mathrm{~mm}$ and $2 l=20$ $\mathrm{mm}$. For the calculations of the energy release rate for the discharging crack model, standard air breakdown field $E_{d}^{c}=3 \mathrm{MV} / \mathrm{m}$ is used. Also, the breakdown strength of PZT ceramics is assumed as $E_{d}=10 \mathrm{MV} / \mathrm{m}^{(21)}$ to predict the dielectric breakdown.

We first consider the cracked piezoelectric ceramics under applied displacement. Table 2 lists the energy release rate for the permeable, impermeable, open and discharging crack models for the rectangular piezoelectric material PCM- 80 under displacement $u_{0}=1 \mu \mathrm{m}$ and electric field $E_{0}=0.1 \mathrm{MV} / \mathrm{m}$ (Case 1). The results obtained by the four domains and the average values (Avg.) are shown, and the values in parentheses are the contribution from the crack interior (see Eq. (36)). Also shown are the results determined from the line integral method. The error in computing the $G$ is reasonable and not important here from the practical point of view. It is found that for the open and discharging crack models, the contribution from the crack interior is negligible under practical loading conditions. Also, it is interesting to note that the energy release rates predicted by the permeable and discharging crack models are not significantly different. In addition, the values determined from the domain and line integral methods are in good agreement. Average $G$ values from the domain integral method are presented in the following figures.

Fig. 3 summarizes the results for the permeable, impermeable, open and discharging cracks normal (Case 1) and parallel (Case 2) to the poling and electric field, and shows the energy release rate $G$ versus electric field $E_{0}$ for the rectangular piezoelectric material PCM80 under $u_{0}=1 \mu \mathrm{m}$. Little differences are observed between the permeable (solid line) and discharging (dot-dashed line) crack models under various electric fields. Under electric fields from 0 to $-0.2 \mathrm{MV} / \mathrm{m}$, polarization switching does not occur. For the piezoelectric material with a crack normal to the poling and electric field (Case 1), the energy release rate $G$ under applied displacement decreases as the positive electric field increases. A negative energy release rate can be induced with positive electric fields in the impermeable and open 


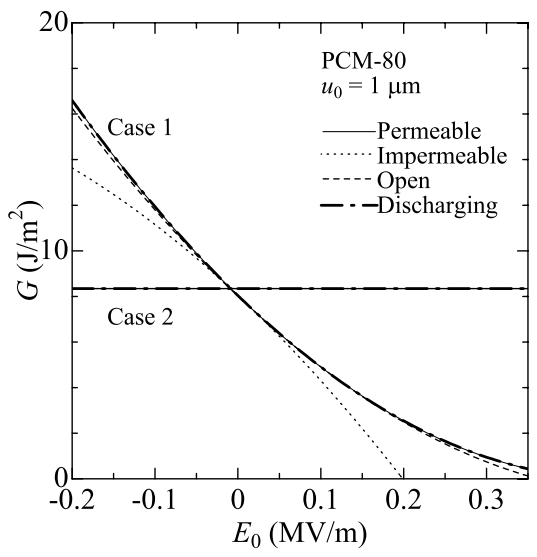

Fig. 3 Energy release rate versus electric field under the displacement $u_{0}=1 \mu \mathrm{m}$ (PCM-80, Cases 1 and 2)

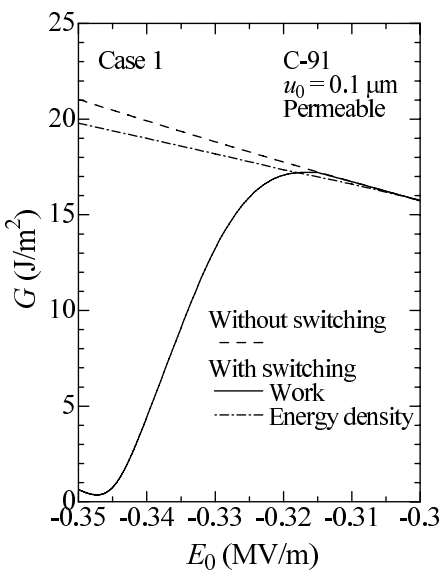

Fig. 4 Energy release rate versus negative electric field under the displacement $u_{0}=$ $0.1 \mu \mathrm{m}(\mathrm{C}-91$, Case 1$)$

crack models. According to the fracture mechanics interpretation, a negative energy release rate would correspond to a crack that could absorb energy due to crack extension. Since this would exclude the fracture in piezoelectric ceramics under electric fields, the parameters for the impermeable and open crack models have questionable physical significance. It is therefore clear that the impermeable and open crack models are not appropriate for a slit crack in piezoelectric ceramics. For the piezoelectric material with a crack parallel to the poling and electric field (Case 2), no difference is observed among four crack models (permeable, impermeable, open and discharging), and the energy release rate is independent of the electric field. Hence, the piezoelectric crack normal to the poling and electric field is important, because the fracture mechanics parameters such as energy release rate strongly depend on the electrical loading condition.

Fig. 4 displays the variation of the $G$ with negative $E_{0}$ for the permeable crack model in the rectangular piezoelectric material C-91 under $u_{0}=0.1 \mu \mathrm{m}$ (Case 1), predicted by the criteria based on the work (Eq.(30)) and energy density (Eq.(31)). Also shown is the result without switching effect. The prediction by the work criterion shows that polarization switching leads to a decrease in $G$ after $E_{0}$ reaches about $-0.315 \mathrm{MV} / \mathrm{m}$. On the other hand, the prediction according to the energy density shows that the value of $G$ deviates gradually from the result without switching effect. The same results are obtained for the discharging crack model (not shown).

Fig. 5 shows the $180^{\circ}$ and $90^{\circ}$ switching zones near the permeable crack tip in the C-91 


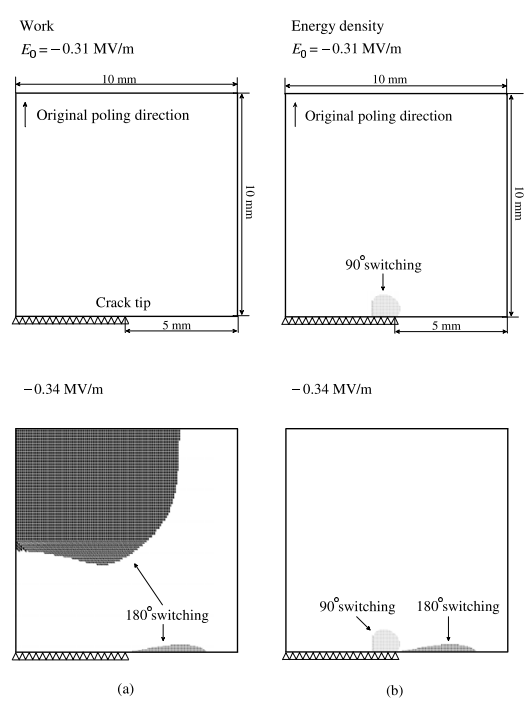

Fig. 5 Polarization switching zone near the permeable crack induced by displacement $u_{0}=0.1 \mu \mathrm{m}$ and electric fields $E_{0}=-0.31,-0.34 \mathrm{MV} / \mathrm{m}$ based on different criteria: (a) work and (b) energy density (C-91, Case 1)

under $u_{0}=0.1 \mu \mathrm{m}$ for $E_{0}=-0.31,-0.34 \mathrm{MV} / \mathrm{m}$ (Case 1), predicted by the criteria based on the (a) work and (b) energy density. The work criterion shows that only the $180^{\circ}$ switching occurs near the crack tip under high negative electric field. If the energy density criterion is used, the region near the crack tip is found to undergo $180^{\circ}$ and $90^{\circ}$ switchings.

Next, we discuss the cracked piezoelectric ceramics under applied stress. Fig. 6 shows the energy release rate $G$ versus electric field $E_{0}$ of the permeable, impermeable, open and discharging crack models in the rectangular piezoelectric material PCM-80 under stress $\sigma_{l}=$ $5.62 \mathrm{MPa}$ (Case 1), corresponding to the uniform displacement $u_{0}=1 \mu \mathrm{m}$ for the uncracked material without the electric field. The results for the permeable and discharging crack models are almost the same. The energy release rates for the impermeable and open crack models become negative under positive and negative electric fields. The negative energy release rate is questionable to be used as a criterion for predicting the crack extension. The energy release rates for the permeable and discharging crack models are positive, and independent of the electric field. In the case under high negative $E_{0}$, the behavior of the $G$ for the permeable crack model is complicated, due to the polarization switching (see Fig. 6(b)). Similar phenomena are also shown for the rectangular piezoelectric material C-91 (no figure shown).

Fig. 7 shows the predicted dielectric breakdown regions near the (a) impermeable and (b) permeable crack tips for the rectangular piezoelectric material PCM-80 under stress $\sigma_{l}=$ 5.62 $\mathrm{MPa}$ and various high positive electric fields $E_{0}$ (Case 1). The dielectric breakdown region increases with an increase of positive $E_{0}$ for the impermeable crack model. However, the energy release rate for the impermeable crack model under high positive $E_{0}$ is negative (see Fig. 6), hence the predicted region is meaningless. In the case of the permeable crack model, the dielectric breakdown does not occur under high positive $E_{0}$ (see Fig. 7(b)). Some previous works dealt with the dielectric breakdown model in cracked piezoelectric ceramics. We recommend the uses of suitable piezoelectric crack model (permeable or discharging) and practical loading conditions.

\section{Conclusions}

The problem of piezoelectric materials with two symmetric edge cracks under tension has numerically been discussed. It was shown that the crack normal to the poling and electric field is important to investigate the piezoelectric fracture mechanics parameters such as energy 


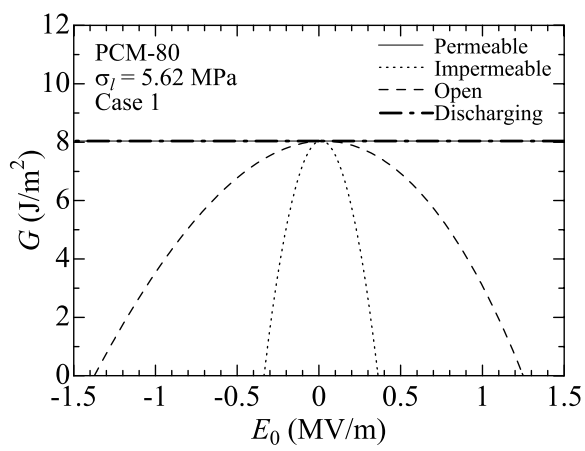

(a)

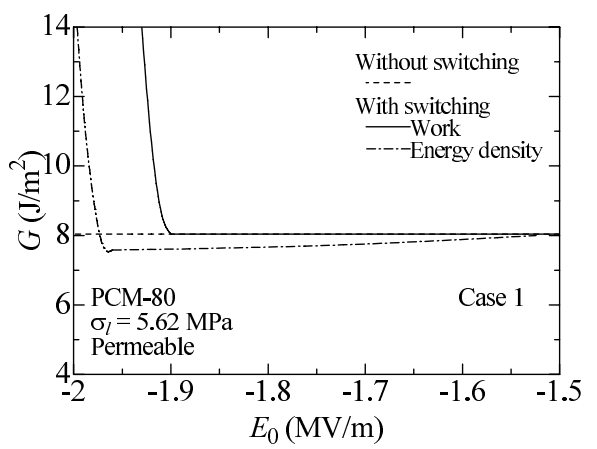

(b)

Fig. 6 Energy release rate versus electric field under the stress $\sigma_{l}=5.62 \mathrm{MPa}$ (PCM80 , Case 1)

release rate. Based on the finite element results of this study, the following conclusions may be drawn:

( 1 ) Piezoelectric crack face boundary conditions strongly affect the electric field effect characteristics of the energy release rate. The energy release rates for the permeable and discharging crack models are identical and always remain positive. On the other hand, the energy release rate criteria for the impermeable and open crack models led to negative values which are unphysical.

(2) If the negative electric field is applied, localized polarization switching occurs due to electromechanical field concentrations near the crack tip, and the switching causes a sudden change in the energy release rate.

( 3 ) If the high positive electric field is applied, dielectric breakdown occurs near the impermeable crack tip. However, the breakdown does not occur near the permeable or discharging crack tip. Different crack models give different results for the dielectric breakdown.

As a remark, the use of suitable crack model (permeable or discharging) is recommended to discuss the piezoelectric crack behavior.

\section{Appendix A}

The changes in spontaneous strains and polarizations for $180^{\circ}$ switching can be expressed as

$$
\begin{aligned}
& \Delta \varepsilon_{x x}=\varepsilon_{x x}^{\mathrm{r}}=0, \Delta \varepsilon_{z z}=\varepsilon_{z z}^{\mathrm{r}}=0, \Delta \varepsilon_{z x}=\varepsilon_{z x}^{\mathrm{r}}=0 \\
& \Delta P_{x}=P_{x}^{\mathrm{r}}=0, \Delta P_{z}=P_{z}^{\mathrm{r}}=-2 P^{\mathrm{s}}
\end{aligned}
$$

For $90^{\circ}$ switching in the $z x$ plane, the changes are

$$
\Delta \varepsilon_{x x}=\varepsilon_{x x}^{\mathrm{r}}=\gamma^{\mathrm{s}}, \Delta \varepsilon_{z z}=\varepsilon_{z z}^{\mathrm{r}}=-\gamma^{\mathrm{s}}, \Delta \varepsilon_{z x}=\varepsilon_{z x}^{\mathrm{r}}=0
$$



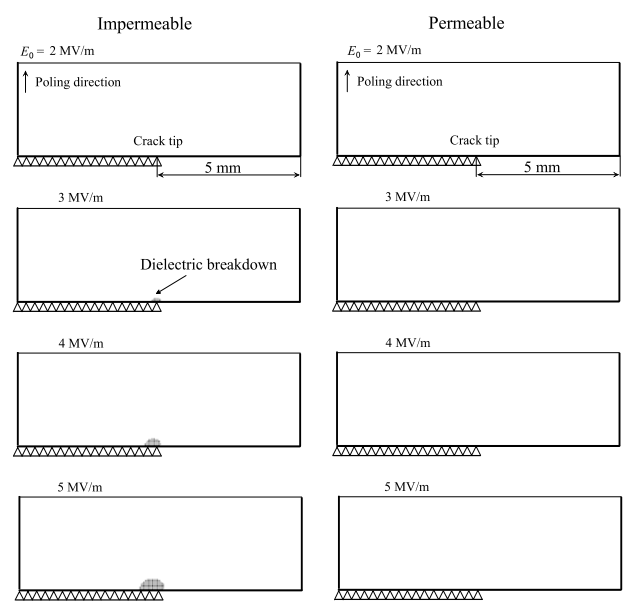

(a)

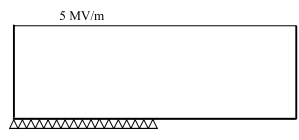

(b)

Fig. 7 Dielectric breakdown region near the (a) impermeable and (b) permeable cracks induced by stress $\sigma_{l}=5.62 \mathrm{MPa}$ and electric fields $E_{0}=2,3,4,5 \mathrm{MV} / \mathrm{m}(\mathrm{PCM}$ 80 , Case 1)

$$
\Delta P_{x}=P_{x}^{\mathrm{r}}= \pm P^{\mathrm{s}}, \Delta P_{z}=P_{z}^{\mathrm{r}}=-P^{\mathrm{s}}
$$

\section{Appendix B}

The constitutive equations (3) and (4) after $180^{\circ}$ switching are

$$
\begin{gathered}
\left\{\begin{array}{c}
\sigma_{x x} \\
\sigma_{z z} \\
\sigma_{z x}
\end{array}\right\}=\left[\begin{array}{ccc}
c_{11} & c_{13} & 0 \\
c_{13} & c_{33} & 0 \\
0 & 0 & c_{44}
\end{array}\right]\left\{\begin{array}{c}
\varepsilon_{x x}-\varepsilon_{x x}^{\mathrm{r}} \\
\varepsilon_{z z}-\varepsilon_{z z}^{\mathrm{r}} \\
2\left(\varepsilon_{z x}-\varepsilon_{z x}^{\mathrm{r}}\right)
\end{array}\right\}+\left[\begin{array}{cc}
0 & e_{31} \\
0 & e_{33} \\
e_{15} & 0
\end{array}\right]\left\{\begin{array}{c}
E_{x} \\
E_{z}
\end{array}\right\} \\
\left\{\begin{array}{c}
D_{x} \\
D_{z}
\end{array}\right\}=-\left[\begin{array}{ccc}
0 & 0 & e_{15} \\
e_{31} & e_{33} & 0
\end{array}\right]\left\{\begin{array}{c}
\varepsilon_{x x}-\varepsilon_{x x}^{\mathrm{r}} \\
\varepsilon_{z z}-\varepsilon_{z z}^{\mathrm{r}} \\
2\left(\varepsilon_{z x}-\varepsilon_{z x}^{\mathrm{r}}\right)
\end{array}\right\} \\
+\left[\begin{array}{cc}
\epsilon_{11} & 0 \\
0 & \epsilon_{33}
\end{array}\right]\left\{\begin{array}{c}
E_{x} \\
E_{z}
\end{array}\right\}+\left\{\begin{array}{c}
P_{x}^{\mathrm{r}} \\
P_{z}^{\mathrm{r}}
\end{array}\right\}
\end{gathered}
$$

The constitutive equations (3) and (4) after $90^{\circ}$ switching become

$$
\begin{gathered}
\left\{\begin{array}{c}
\sigma_{x x} \\
\sigma_{z z} \\
\sigma_{z x}
\end{array}\right\}=\left[\begin{array}{ccc}
c_{11} & c_{13} & 0 \\
c_{13} & c_{33} & 0 \\
0 & 0 & c_{44}
\end{array}\right]\left\{\begin{array}{c}
\varepsilon_{x x}-\varepsilon_{x x}^{\mathrm{r}} \\
\varepsilon_{z z}-\varepsilon_{z z}^{\mathrm{r}} \\
2\left(\varepsilon_{z x}-\varepsilon_{z x}^{\mathrm{r}}\right)
\end{array}\right\} \mp\left[\begin{array}{cc}
e_{33} & 0 \\
e_{31} & 0 \\
0 & e_{15}
\end{array}\right]\left\{\begin{array}{c}
E_{x} \\
E_{z}
\end{array}\right\} \\
\left\{\begin{array}{c}
D_{x} \\
D_{z}
\end{array}\right\}= \pm\left[\begin{array}{ccc}
e_{33} & e_{31} & 0 \\
0 & 0 & e_{15}
\end{array}\right]\left\{\begin{array}{c}
\varepsilon_{x x}-\varepsilon_{x x}^{\mathrm{r}} \\
\varepsilon_{z z}-\varepsilon_{z z}^{\mathrm{r}} \\
2\left(\varepsilon_{z x}-\varepsilon_{z x}^{\mathrm{r}}\right)
\end{array}\right\} \\
+\left[\begin{array}{cc}
0 & \epsilon_{33} \\
\epsilon_{11} & 0
\end{array}\right]\left\{\begin{array}{c}
E_{x} \\
E_{z}
\end{array}\right\}+\left\{\begin{array}{c}
P_{x}^{\mathrm{r}} \\
P_{z}^{\mathrm{r}}
\end{array}\right\}
\end{gathered}
$$

\section{References}

( 1 ) Shindo, Y., Ozawa, E., Nowacki, J. P., Singular stress and electric fields of a cracked piezoelectric strip, International Journal of Applied Electromagnetics in Materials, Vol. 1, No. 1 (1990), pp. 77-87. 
( 2 ) Sosa, H. A., On the fracture mechanics of piezoelectric solids, International Journal of Solids and Structures, Vol. 29, No. 21 (1992), pp. 2613-2622.

( 3 ) Pak, Y. E., Linear electro-elastic fracture mechanics of piezoelectric materials, International Journal of Fracture, Vol. 54 No. 1 (1992), pp. 79-100.

( 4 ) Hao T.-H., Shen Z.-Y., A new electric boundary condition of electric fracture mechanics and its applications, Engineering Fracture Mechanics, Vol. 47, No. 6 (1994), pp. 793 802.

( 5 ) Landis C. M., Energetically consistent boundary conditions for electromechanical fracture, International Journal of Solids and Structures, Vol. 41, No. 22-23 (2004), pp. 6291-6315.

( 6 ) Shindo, Y., Narita, F., Hirama M., Effect of the electrical boundary condition at the crack face on the mode I energy release rate in piezoelectric ceramics, Applied Physics Letters, Vol. 94, No. 8 (2009), 081902.

( 7 ) Fang, D. N., Sun, C. T., Experimental study of fatigue and fracture of piezoceramics, Mechanics of Electromagnetic Material Systems and Structures, Edited by Shindo, Y., WIT Press, 2003, pp. 103-114.

( 8 ) Shindo, Y., Narita, F., Mikami, M., Double torsion testing and finite element analysis for determining the electric fracture properties of piezoelectric ceramics, Journal of Applied Physics, Vol. 97, No. 11 (2005), 114109.

( 9 ) Shindo, Y., Narita, F., Horiguchi, K., Magara, Y., Yoshida M., Electric fracture and polarization switching properties of piezoelectric ceramic PZT studied by the modified small punch test, Acta Materialia, Vol. 51, No. 16 (2003), pp. 4773-4782.

(10) Gao, H., Zhang, T.-Y., Tong, P., Local and global energy release rates for an electrically yielded crack in a piezoelectric ceramic, Journal of the Mechanics and Physics of Solids, Vol. 45, No. 4 (1997), pp. 491-510.

(11) Zhang, T.-Y., Zhao, M.-H., Gao, C.-F., The strip dielectric breakdown model, International Journal of Engineering Science, Vol. 132, No. 3-4 (2005), pp. 311-327.

(12) Fan, C.-Y., Zhao, M.-H., Zhou, Y.-H., Numerical solution of polarization saturation/dielectric breakdown model in 2D finite piezoelectric media, Journal of the Mechanics and Physics of Solids, Vol. 57, No. 9 (2009), pp. 1527-1544.

(13) Narita, F., Shindo, Y., Horiguchi, K., Electroelastic fracture mechanics of piezoelectric ceramics, Mechanics of Electromagnetic Material Systems and Structures, Edited by Shindo, Y., WIT Press, 2003, pp. 89-101.

(14) Hwang, S. C., Lynch, C. S., McMeeking, R. M., Ferroelectric/ferroelastic interactions and a polarization switching model, Acta Metallurgica et Materialia, Vol. 43, No. 5 (1995), pp. 2073-2084.

(15) Sun, C. T., Achuthan, A., Domain-switching criteria for ferroelectric materials subjected to electrical and mechanical loads, Journal of the American Ceramic Society, Vol. 87, No. 3 (2004), pp. 395-400.

(16) McMeeking, R. M., The energy release rate for a Griffith crack in a piezoelectric material, Engineering Fracture Mechanics, Vol. 71, No. 7-8 (2004), pp. 1149-1163.

(17) Li, F. Z., Shih, C. F., Needleman, A., A comparison of methods for calculating energy release rates, Engineering Fracture Mechanics, Vol. 21, No. 2 (1985), pp. 405-421.

(18) McMeeking, R. M., Crack tip energy release rate for a piezoelectric compact tension specimen, Engineering Fracture Mechanics, Vol. 64, No. 2 (1999), pp. 217-244.

(19) Shindo, Y., Yoshida, M., Narita, F., Horiguchi, K., Electroelastic field concentrations ahead of electrodes in multilayer piezoelectric actuators: experiment and finite element simulation, Journal of the Mechanics and Physics of Solids, Vol. 52, No. 5 (2004), pp. 1109-1124.

(20) Narita, F., Shindo, Y., Hayashi, K., Bending and polarization switching of piezoelectric laminated actuators under electromechanical loading, Computers $\mathcal{E}$ Structures, Vol. 83, No. 15-16 (2005), pp. 1164-1170. 
(21) Tani, T., Asai, M., Takatori K., Kamiya, N., Evaluation of dielectric strength and breakdown behavior for Sr-, Nb-doped PZT ceramics with various shapes of electrodes, Journal of the Ceramic Society of Japan, Vol. 105, No. 4 (1997), pp. 308-311. 